\title{
Influence of the Fluoride Atoms Doping on the FeSe Superconductor
}

\author{
A. D. Bortolozo ${ }^{1,2}$, A. D. Gueiros ${ }^{1}$, L. M. S. Alves ${ }^{1}$, C. A. M. dos Santos ${ }^{1}$ \\ ${ }^{1}$ Departamento de Engenharia de Materiais, Escola de Engenharia de Lorena-USP, Lorena, Brazil; ${ }^{2}$ Universidade Federal de Itajubá, \\ Campus de Itabira, Itabira, Brazil. \\ Email: ausdinirbortolozo@unifei.edu.br
}

Received March $29^{\text {th }}, 2012$; revised May $2^{\text {nd }}, 2012$; accepted July $3^{\text {rd }}, 2012$

\begin{abstract}
It is reported the influence of the interstitial atoms doping on the FeSe superconductor. Polycrystalline samples with $\mathrm{FeSeF}_{x}$ and $\mathrm{FeSeB}_{x}$ nominal compositions were prepared by solid state reaction. An enhancement of the superconducting transition temperature was observed in the temperature dependence of the electrical resistivity curve to the $\mathrm{FeSeF}_{0.015}$ sample. $\mathrm{R}(\mathrm{T})$ data display superconducting behavior close to $12 \mathrm{~K}$. The $\mathrm{T}_{\mathrm{c}}$ increased with $\mathrm{F}$ doping by up to $50 \%$. In contrast, boron doping no change the superconducting properties of the FeSe compound. As the $\mathrm{FeSe}_{1-x} \mathrm{Te}_{x}$ system the fluoride doping introduce a negative chemical pressure in the FeSe superconductor. This fact suggests that fluoride doping have changed the electronic properties of the FeSe phase.
\end{abstract}

Keywords: Superconductivity; Pnictides; Interstitial

\section{Introduction}

Since the surprisingly discovery of superconductivity in high temperature of $55 \mathrm{~K}$ to the fluorine-doped rare-earth iron oxyarsenides, $\mathrm{REFeAs}_{1-x} \mathrm{~F}_{x}$, many research has been made on new superconducting materials [1-4]. The new materials class has $\mathrm{Fe}$ and share a common structural feature. In 2008, Hsu et al. [5] reported the superconductivity at $8 \mathrm{~K}$ in PbO-prototype FeSe compound knows as "11" family. FeSe system is one of the most attracting systems, because it has the simplest crystal structure among the ironbased superconductors $[5,6]$. McQueen has been shown that superconductivity in $\mathrm{Fe}_{1+\delta} \mathrm{Se}$ is destroyed by very small changes in stoichiometric [7]. They also showed that nonsuperconducting $\mathrm{Fe}_{1+\delta} \mathrm{Se}$ is not magnetically ordered down to $5 \mathrm{~K}$ and it is destroyed by very small changes in stoichiometry. Then, immediate instability against an ordered magnetic state should not be considered as intrinsic characteristic [7].

While a superconducting transition temperature $T_{c}$ of FeSe at ambient pressure is $\sim 8 \mathrm{~K}$, the $\mathrm{T}_{\mathrm{c}}$ has been raised to $27 \mathrm{~K}$ with the application of pressure [8-10]. Compared with the layered FeAs systems, FeSe has not only the same planar sublattices but also displays structure and magnetic instability [11-14]. Within the family of "11" binary iron chalcogenides the superconducting state exists over a wide range of Te-doping in the $\mathrm{Fe}(\mathrm{Se}, \mathrm{Te})$ solid solution with maximum $T_{c}$ of $15 \mathrm{~K}$ [15-22]. These substitutions generate chemical pressures, because $\mathrm{S}, \mathrm{Se}$ and
Te have the same valence and the different ionic radius. Considering the huge positive pressure effect on $\mathrm{T}_{c}$, compressing the lattice seems to be effective to enhance $T_{c}$. However the increase of $\mathrm{T}_{\mathrm{c}}$ in $\mathrm{FeSe}_{1-x} \mathrm{~S}_{x}$, which is chemically pressured $\mathrm{FeSe}$, is only $2 \mathrm{~K}$. On the other hand, the increase of $\mathrm{T}_{\mathrm{c}}$ in $\mathrm{FeSe}_{1-x} \mathrm{Te}_{x}$, which is applied a negative chemical pressure, is the lager value of $5 \mathrm{~K}$. In fact, the physical pressure and chemical pressure effects are not equivalent in FeSe system [15-22]. Here we report the influence of interstitial doping on the FeSe superconductor. We found that the $\mathrm{FeSe}+0.5 \% \mathrm{FeF}_{3}$ shows superconducting transition like $\mathrm{Fe}(\mathrm{SeTe})$ solid solution. On the other hand, the low content boron atoms doping do not change the electric and magnetic properties of FeSe superconductor.

\section{Experimental Procedure}

$\mathrm{FeSeF}_{x}$ were prepared using mixtures of appropriate amount of high purity $\mathrm{Fe}, \mathrm{Se}$, and $x \% \mathrm{FeF}_{3}(x=0.1,0.3$, and 0.5$)$ powders. The mixtures were compacted in square shape of $10 \times 10 \mathrm{~mm}^{2}$ and $2 \mathrm{~mm}$ thick, sealed in a quartz ampoule, and placed inside a box furnace model Lindberg/ Blue $\mathrm{M}$ furnace at $1000^{\circ} \mathrm{C}$ for 10 hours, and cooled at $400^{\circ} \mathrm{C}$ and, then kept for 3 days. After this heat treatment, the samples were ground and homogenized in agate mortar, compacted again in the same dimensions mentioned before, and sintered at $400^{\circ} \mathrm{C}$ for 3 days. After this additional heat treatment, the samples were submitted to a rapid 
quenching in water in order to trap the tetragonal phase. All samples were characterized by X-ray powder diffraction in a Shimadzu diffractometer (model XRD 6000) using $\mathrm{Ni}$ filter $\mathrm{CuK} \alpha$ radiation. The simulation of the structure and refinement of the lattice parameters were carried out by using both the Powder Cell software and the Rietveld method. The simulation of the crystal structure was based on the literature data and the results were compared with the experimental diffractograms. The electrical resistance as a function of temperature, $\mathrm{R}(\mathrm{T})$, were performed by conventional four-point probe method in the temperature interval between 2.0 and $300 \mathrm{~K}$. In order to remove the thermal power effects arising in the silver-soldered samples terminal, the measurements were done by applying an alternating polarity of the dc current and taking the average voltage between the two measurements.

\section{Results and Discussions}

$\mathrm{X}$-ray powder diffraction data at room temperature for the $\mathrm{FeSe}+x \% \mathrm{FeF}_{3}(x=0.1 ; 0.3$ and 0.5$)$ samples are displayed in Figure1. All peaks can be indexed based on tetragonal structure space group $\mathrm{P} 4 / \mathrm{nmm}$, with lattice parameters $\mathrm{a}=3.36 \AA$ and $\mathrm{c}=5.52 \AA$. These results reveal that $\mathrm{F}$ atoms are been solubilized within the crystalline structure of the space group $\mathrm{P} 4 / \mathrm{nmm}$.

In order to determine what site fluoride doping is occupied the refinement of X-ray data was made. The initial analysis suggests that $\mathrm{F}$ atoms could occupy the " $4 \mathrm{~d}$ " Wyckoff position. While the increase $\mathrm{F}$ content form nominally $0.3 \%$ to $0.5 \%$ leads to relatively small chances in the lattice parameters. The anisotropic expansion of the crystalline structure can be observed on the (200) and (003) reflection. It is possible to observed in the (003) reflection that there is not significantly expansion/contraction of the lattice parameter. On the other hand the (200) reflection the small change could be related.

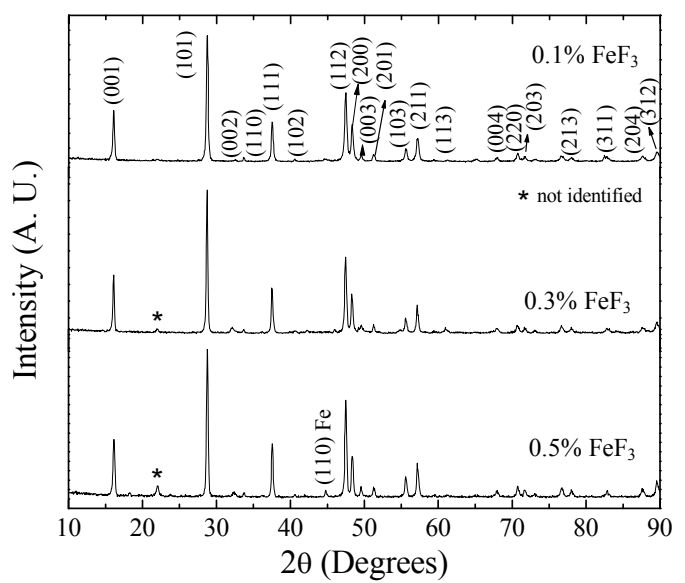

Figure 1. X-ray diffraction for the $\mathrm{FeSe}+x \% \mathrm{FeF}_{3}$ showing that the fluoride does not collapse the $\mathrm{PbO}$ prototype structure.
In order to study the transport properties of the $\mathrm{FeSe}+$ $x \% \mathrm{FeF}_{3}$ samples, electrical resistance measurements as a function of temperature have been performed. $\mathrm{R}(\mathrm{T})$ curve for the $\mathrm{FeSe}+0.1 \% \mathrm{FeF}_{3}$ reveals a typical metallic behavior between 15 and $150 \mathrm{~K}$ (Figure 2).

The superconducting transition at $10 \mathrm{~K}$ with transition broadening of $3 \mathrm{~K}$ can be observed in the measurement. This fact shows the fluoride doping does not destroy the superconductivity of the FeSe superconductor. On the other hand, the fluoride doping increases the superconducting temperature. In the inset of the Figure 2 is displayed the measurement between $4.0 \mathrm{~K}$ and $25.0 \mathrm{~K}$ with different electrical currents.

In the Figure 3 the $\mathrm{R}(\mathrm{T})$ curve for the $\mathrm{FeSe}+0.3 \%$ $\mathrm{FeF}_{3}$ reveals like metallic behavior between 15 and 300 $\mathrm{K}$. On the other hand, the superconducting transition is close to $11.2 \mathrm{~K}$. This fact suggests the fluoride is changing the transport properties of the FeSe superconductor.

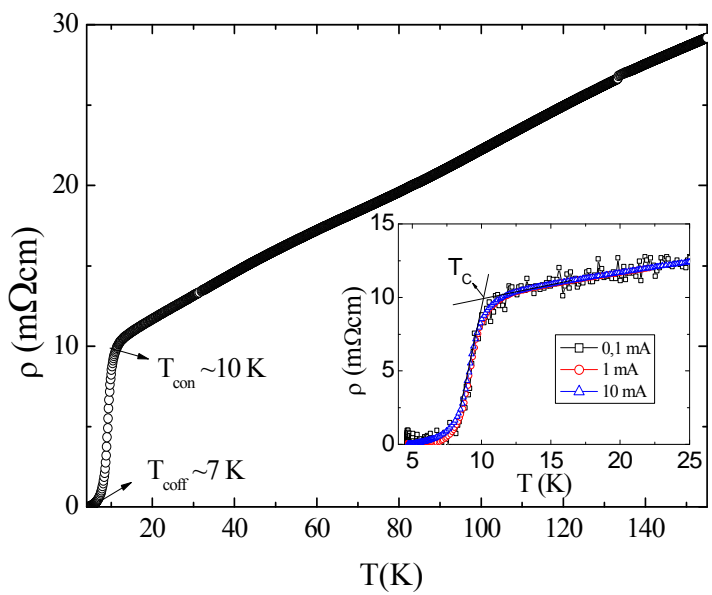

Figure 2. Electrical resistance as a function of temperature from 4 to $150 \mathrm{~K}$ to the $\mathrm{FeSe}+0.1 \% \mathrm{FeF}_{3}$ shows the superconducting transition at $10 \mathrm{~K}$. The normal state is an electron- phonon scattering.

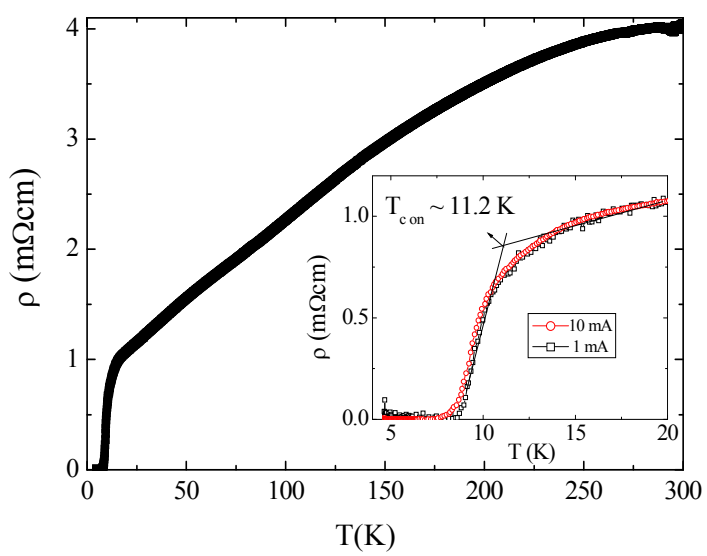

Figure 3. Electrical resistivity as a function of temperature for the $\mathrm{FeSe}+0.3 \% \mathrm{FeF}_{3}$ sample. Inset displays the superconducting transition starting at $\sim 11.2 \mathrm{~K}$. 
In Figure 4 is shown the $\mathrm{R}(\mathrm{T})$ for the $\mathrm{FeSe}+0.5 \%$ $\mathrm{FeF}_{3}$. The $\mathrm{FeSe}+0.5 \% \mathrm{FeF}_{3}$ compound superconducts at $13 \mathrm{~K}$ (onset temperature). This transition temperature is compared to $\mathrm{FeSe}_{0.5} \mathrm{Te}_{0.5}$. The transition broadening is due to impurity in the $\mathrm{FeSe}+0.5 \% \mathrm{FeF}_{3}$ as is possible to observed in the X-ray diffraction data.

Figure 5 show the normalized electrical resistance as a function of temperature to the $\mathrm{FeSeF}_{x}$. The $\mathrm{R}(\mathrm{T})$ data is clear the $\mathrm{F}$ doping is changed the electrical behavior of the FeSe superconductor.

The low content boron atoms doping do not collapse the crystalline structure nor change the electrical and magnetic properties of the FeSe superconductor.

\section{Conclusion}

This work has investigated the effect of fluoride doping in the FeSe superconductor, which exhibits the simplest crystal structure among the iron-based superconductors.

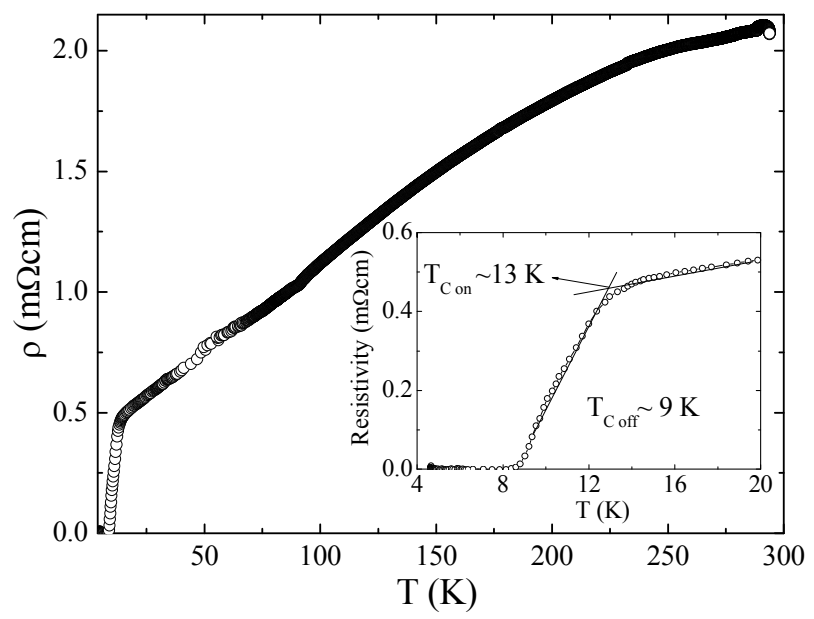

Figure 4. Electrical resistivity as a function of temperature

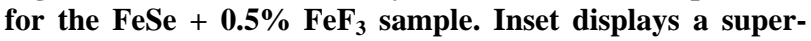
conducting transition starting at $\sim 13 \mathrm{~K}$.

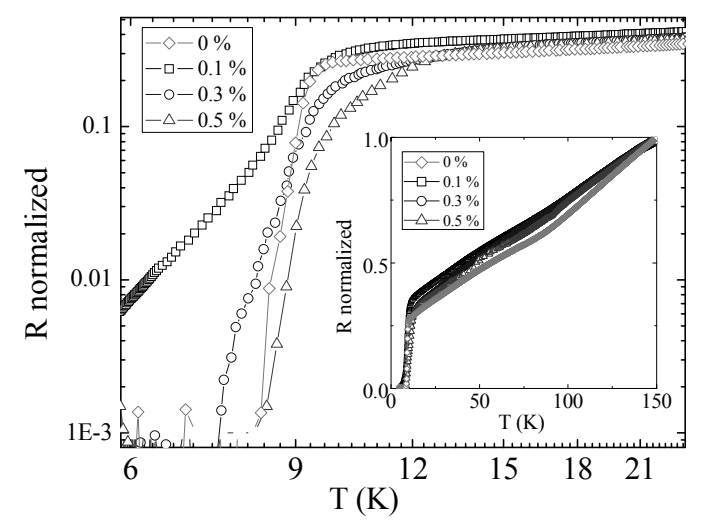

Figure 5. Electrical resistance normalized as a function of temperature to the $\mathrm{FeSeF}_{x}$ compounds. The FeSe $+0.5 \%$ $\mathrm{FeF}_{3}$ compound superconducts at $13 \mathrm{~K}$ (onset temperature). This transition temperature is compared to $\mathrm{FeSe}_{0.5} \mathrm{Te}_{0.5}$.
The results show is possible to dope the FeSe superconductor with the interstitial atoms. The boron doping not changes the superconducting properties of the FeSe. On the other hand the fluoride doping applied a negative chemical pressure in the FeSe superconductor which increases the $\mathrm{T}_{\mathrm{c}}$ like in the $\mathrm{FeSe}_{1-x} \mathrm{Te}_{x}$ system.

\section{Acknowledgements}

We are grateful for the helpful discussion with Prof. Antonio Jefferson S Machado. This work is based upon supported by FAPESP (Grants $n$ 2009/00610-8) and by FAPEMIG (Grants n PRI-00049-12).

\section{REFERENCES}

[1] Y. Kamihara, T. Watanabe, M. Hirano and H. Hosono, "Iron-Based Layered Superconductor $\mathrm{La}\left[\mathrm{O}_{1-x} \mathrm{~F}_{x}\right] \mathrm{FeAs}$ $(x=0.05-0.12)$ with $\mathrm{T}_{\mathrm{c}}=26 \mathrm{~K}$," Journal of the American Chemical Society, Vol. 130, No. 11, 2008, pp. 3296 3297.doi:10.1021/ja800073m

[2] X. H. Chen, T. Wu, G. Wu, R. H. Liu, H. Chen and D. F. Fang, "Superconductivity at $43 \mathrm{~K}$ in $\mathrm{SmFeAsO}_{1-x} \mathrm{~F}_{x}$," Nature, Vol. 453, No. 5, 2008, pp. 761-762. doi:10.1038/nature07045

[3] R. H. Liu, G. Wu, T. Wu, D. F. Fang, H. Chen, S. Y. Li, K. Liu, Y. L. Xie, X. F. Wang, R. L. Yang, L. Ding, C. He, D. L. Feng and X. H. Chen, "Anomalous Transport Properties and Phase Diagram of the FeAs-Based $\mathrm{SmFeAsO}_{1-x} \mathrm{~F}_{x}$ Superconductors," Physical Review Letters, Vol. 101, No. 8, 2008, Article ID: 087001. doi:10.1103/PhysRevLett.101.087001

[4] Y. Takabayashi, M. T. McDonald, D. Papanikolaou, S. Margadonna, G. Wu, R. H. Liu, X. H. Chen and K. Prassides, "Doping Dependence of the Pressure Response of $\mathrm{T}_{\mathrm{c}}$ in the $\mathrm{SmO}_{1-x} \mathrm{~F}_{x} \mathrm{FeAs}$ Superconductors," Journal of the American Chemical Society, Vol. 130, No. 9, 2008, pp. 9242-9243. doi:10.1021/ja8036838

[5] F. C. Hsu, J. Y. Luo, K. W. Yeh, T. K. Chen, T. W. Huang, P. M. Wu, Y. C. Lee, Y. L. Huang, Y. Y. Chu, D. C. Yan and M. K. Wu, "Superconductivity in the PbOType Structure Alpha-FeSe," Proceedings of the National Academy of Sciences of the United States of America, Vol. 105, No. 38, 2008, pp. 14262-14264. doi:10.1073/pnas.0807325105

[6] C. C. Chang, C. H. Wang, M. H. Wen, Y. R. Wu, Y. T. Hsieh and M. K. Wu, "Superconductivity in PbO-Type Tetragonal FeSe Nanoparticles," Solid State Communications, Vol. 152, No. 8, 2012, pp. 649-652. doi:10.1016/j.ssc.2012.01.030

[7] T. M. McQueen, Q. Huang, V. Ksenofontov, C. Felser, Q. $\mathrm{Xu}$, H. Zandbergen, Y. S. Hor, J. Allred, A. J. Williams, D. Qu, J. Checkelsky, N. P. Ong and R. J. Cava, "Extreme Sensitivity of Superconductivity to Stoichiometry in $\mathrm{Fe}_{1+\delta}$ Se," Physical Review B, Vol. 1, No. 79, 2009, Article ID: 014522. doi:10.1103/PhysRevB.79.014522

[8] Y. Mizuguchi, F. Tomioka, S. Tsuda, T. Yamaguchi and Y. Takano, "Superconductivity at $27 \mathrm{~K}$ in Tetragonal FeSe under High Pressure," Applied Physics Letters, Vol. 
93, No. 15, 2008, Article ID: 152505. doi:10.1063/1.3000616

[9] T. Imai, K. Ahilan, F. L. Ning, T. M. McQueen and R. J. Cava, "Why Does Undoped FeSe Become a High- $\mathrm{T}_{\mathrm{c}} \mathrm{Su}-$ perconductor under Pressure?" Physical Review Letters, Vol. 102, No. 17, 2009, Article ID: 177005. doi:10.1103/PhysRevLett.102.177005

[10] J N. Millican, D. Phelan, E. L. Thomas, J. B. Leão and E. Carpenter, "Pressure-Induced Effects on the Structure of the FeSe Superconductor," Solid State Communications, Vol. 149, No. 17-18, 2009, pp. 707-710. doi:10.1016/j.ssc.2009.02.011

[11] G. Rahman, I. G. Kim and A. J Freeman, "Ab Initio Prediction of Pressure-Induced Structural Phase Transition of Superconducting FeSe," Journal of Physics: Condensed Matter, Vol. 24, No. 9, 2012, Article ID: 095502. doi:10.1088/0953-8984/24/9/095502

[12] M. H. Fang, H. M Pham, B. Qian, T. J. Liu, E. K. Vehstedt, Y. Liu, L. Spinu and A. Q. Mao, "Superconductivity Close to Magnetic Instability in

$\mathrm{Fe}\left(\mathrm{Se}_{1-x} \mathrm{Te}_{x}\right)_{0.82}$, , Physical Review B, Vol. 78, No. 22, 2008, Article ID: 224503. doi:10.1103/PhysRevB.78.224503

[13] H. Kotegawa, S. Masaki, Y. Awai, H. Tou, Y. Mizuguchi and Y. Takano, "Evidence for Unconventional Superconductivity in Arsenic-Free Iron-Based Superconductor FeSe: A ${ }^{77}$ Se-NMR Study," Journal of the Physical Society of Japan, Vol. 77, 2008, Article ID: 113703. doi:10.1143/JPSJ.77.113703

[14] G. E. Grechnev, A. S. Panfilov, A. V. Fedorchenko, V. A. Desnenko, S. L. Gnatchenko, V. Tsurkan, J. Deisenhofer, A. Loidl, D. A. Chareev, O. S. Volkova and A. N. Vasiliev, "Magnetic Properties of Novel FeSe(Te) Superconductors," Journal of Magnetism and Magnetic Materials, Vol. 324, No. 21, 2012, pp. 3460-3463.

[15] K. W. Yeh, T. T. W. Huang, Y. L. Huang, T. K. Chen, F. C. Hsu, P. M. Wu, Y. C. Lee, Y.Y. Chu, C. L. Chen, J. Y. Luo, D. C. Yan and M. K. Wu, "Tellurium Substitution Effect on Superconductivity of the $\alpha$-Phase Iron Se- lenide," Europhysics Letters, Vol. 84, No. 3, 2008, Article ID: 37002 . doi:10.1209/0295-5075/84/37002

[16] B. C. Sales, A. S. Sefat, M. A. McGuire, R. Y. Jin and D. Mandrus, "Bulk Superconductivity at $14 \mathrm{~K}$ in Single Crystals of $\mathrm{Fe}_{1+y} \mathrm{Te}_{x} \mathrm{Se}_{1-x}$," Physical Review B, Vol. 79, No. 9, 2009, Article ID: 094521. doi:10.1103/PhysRevB.79.094521

[17] T. L. Xia, D. Hou, S. C. Zhao, A. M. Zhang, G. F. Chen, J. L. Luo, N. L. Wang, J. H. Wei, Z.-Y. Lu and Q. M. Zhang, "Raman Phonons of $\alpha$-FeTe and $\mathrm{Fe}_{1.03} \mathrm{Se}_{0.3} \mathrm{Te}_{0.7}$ Single Crystals," Physical Review B, Vol. 79, No. 14, 2009, Article ID: 140510 doi:10.1103/PhysRevB.79.140510

[18] K. W. Yeh, H. C. Hsu, T. W. Huang, P. M. Wu, Y. L. Huang, T. K. Chen, J. Y. Luo and M. K. Wu, "Se and Te Doping Study of the FeSe Superconductors," Journal of the Physical Society of Japan, Vol. 77, Suppl. C, 2008, pp. 19-22.

[19] Y. Mizuguchi, F. Tomioka, S. Tsuda, T. Yamaguchi and Y. Takano, "Substitution Effects on FeSe Superconductor," Journal of the Physical Society of Japan, Vol. 78, No. 7, 2009, Article ID: 074712. doi:10.1143/JPSJ.78.074712

[20] S. Li, C. de la Cruz, Q. Huang, Y. Chen, J. W. Lynn, J. Hu, Y. L. Huang, F. C. Hsu, K. W. Yeh, M. K. Wu and P. Dai, "First-Order Magnetic and Structural Phase Transitions in $\mathrm{Fe}_{1+y} \mathrm{Se}_{x} \mathrm{Te}_{1-x}$," Physical Review B, Vol. 79, No. 5, 2009, Article ID: 054503. doi:10.1103/PhysRevB.79.054503

[21] K. W. Yeh, C. T. Ke, T. W. Huang, T. K. Chen, Y. L. Huang, P. M. Wu and M. K. Wu, "Superconducting $\mathrm{FeSe}_{1-x} \mathrm{Te}_{x}$ Single Crystals Grown by Optical ZoneMelting Technique," Crystal Growth \& Design, Vol. 9, No. 11, 2009, pp. 4847-4851. doi:10.1021/cg900675e

[22] R. Viennois, E. Giannini, D. van der Marel and R. Černý, "Effect of Fe Excess on Structural, Magnetic and Superconducting Properties of Single-Crystalline $\mathrm{Fe}_{1+x} \mathrm{Te}_{1-y} \mathrm{Se}_{y}$," Journal of Solid State Chemistry, Vol. 183, No. 4, 2010, pp. 769-775. doi:10.1016/j.jssc.2010.01.024 